\title{
BMJ Open PROSPECTIV - a pilot trial of a nurse-led psychoeducational intervention delivered in primary care to prostate cancer survivors: study protocol for a randomised controlled trial
}

\author{
Eila Watson, ${ }^{1}$ Peter Rose ${ }^{2}$ Emma Frith, ${ }^{1}$ Freddie Hamdy, ${ }^{3}$ David Neal, ${ }^{4}$ \\ Christof Kastner, ${ }^{4}$ Simon Russell, ${ }^{4}$ Fiona M Walter, ${ }^{5}$ Sara Faithfull, ${ }^{6}$ \\ Jane Wolstenholme, ${ }^{7}$ Rafael Perera, ${ }^{2}$ David Weller, ${ }^{8}$ Christine Campbell, ${ }^{8}$ \\ Clare Wilkinson, ${ }^{9}$ Richard Neal, ${ }^{9}$ Prasanna Sooriakumaran, ${ }^{3}$ Hugh Butcher, ${ }^{10}$ \\ Mike Matthews ${ }^{10}$
}

To cite: Watson E, Rose $P$, Frith E, et al. PROSPECTIVa pilot trial of a nurse-led psychoeducational intervention delivered in primary care to prostate cancer survivors: study protocol for a randomised controlled trial. BMJ Open 2014;4:e005186.

doi:10.1136/bmjopen-2014005186

- Prepublication history and additional material is available. To view please visit the journal (http://dx.doi.org/ 10.1136/bmjopen-2014005186).

Received 4 March 2014 Accepted 1 May 2014

CrossMark

For numbered affiliations see end of article.

Correspondence to Professor Eila Watson; ewatson@brookes.ac.uk

\section{ABSTRACT}

Background: Prostate cancer survivors can experience physical, sexual, psychological and emotional problems, and there is evidence that current follow-up practices fail to meet these men's needs. Studies show that secondary and primary care physicians see a greater role for primary care in delivering follow-up, and that primary care-led follow-up is acceptable to men with prostate cancer.

Methods and analysis: A two-phase study with target population being men who are 9-24 months from diagnosis. Phase 1 questionnaire aims to recruit 300 men and measure prostate-related quality of life and unmet needs. Men experiencing problems with urinary, bowel, sexual or hormonal function will be eligible for phase 2, a pilot trial of a primary care nurse-led psychoeducational intervention. Consenting eligible participants will be randomised either to intervention plus usual care, or usual care alone (40 men in each arm). The intervention, based on a self-management approach, underpinned by Bandura's Social Cognitive Theory, will provide advice and support tailored to these men's needs and address any problems they are experiencing. Telephone follow-up will take place at 6 months. Study outcomes will be measured by a questionnaire at 7 months. Phase 1 will allow us to estimate the prevalence of urinary, sexual, bowel and hormone-related problems in prostate cancer survivors and the level of unmet needs. 'Usual care' will also be documented. Phase 2 will provide information on recruitment and retention, acceptability of the intervention/outcome measures, effect sizes of the intervention and cost-effectiveness data, which is required to inform development of a larger, phase 3 randomised controlled trial. The main outcome of interest is change in prostate-cancer-related quality of life. Methodological issues will also be addressed.

Ethics and dissemination: Ethics approval has been gained (Oxford REC A 12/SC/0500). Findings will be disseminated in peer-reviewed journals, at conferences, through user networks and relevant clinical groups.

\section{Strengths and limitations of this study}

- The study addresses a recognised gap in the care of men with prostate cancer.

- It promotes a model of care consistent with that being recommended by the UK National Cancer Survivorship Initiative.

- The intervention is evidence based and theoretically informed. Development involved a multidisciplinary team, including user representatives.

- Participants were recruited from only two regional centres for this pilot trial, which may affect generalisability of the findings.

- As intervention delivery will span an 18-month period, retention of trained study nurses may be an issue.

- Other health initiatives may occur for this patient group over the study period which could affect the impact of our intervention.

Trial registration number: ISRCTN 97242511.

\section{BACKGROUND}

Prostate cancer is the most common cancer in men in the UK, with more than 40000 cases diagnosed in 2010. Incidence of the disease has been rising steadily over the past 10 years. Survival rates are also increasing and for the period 2005-2009 the 5-year survival rate was $81.4 \% .^{1}$ There is therefore, a large and growing number of men living with prostate cancer in the UK-currently around 250000 , predicted to rise to 831000 by $2040 .^{2}$

Previous studies have demonstrated that prostate cancer survivors can experience physical, sexual and psychological problems 
following diagnosis and treatment, and that current follow-up practices are failing to meet these needs. ${ }^{3-11}$ For example, one US study ${ }^{8}$ which assessed quality of life for men with localised disease over 24 months found that at 6 months, sexual function was a problem for $59 \%$ of surgical patients, $30 \%$ of patients who had external beam radiotherapy and $34 \%$ of patients who had brachytherapy. At 24 months these figures were 43\%, 37\% and 30\%, respectively. Among men receiving radiotherapy, adjuvant hormone therapy was associated with poorer sexual function. Urinary problems at 2 months following treatment were reported for $29 \%$ of surgical patients, $30 \%$ of external beam radiotherapy patients and 39\% of brachytherapy patients. By 24 months these proportions were $7 \%$, $11 \%$ and $16 \%$, respectively. An Australian populationbased study reported that men in all treatment groups had worse sexual function than a control population at 1 , 2 and 3 years. All treatment groups also reported greater urinary 'bother'. ${ }^{9}$ In the UK, Ream $e t$ al conducted a survey with men diagnosed 3-24 months prior which showed that the areas of greatest need reported by these men were psychological distress, sexuality-related issues and the management of ongoing urinary symptoms. A qualitative study we conducted also highlighted the importance of unmet psychosexual needs and some of the shortcomings of existing follow-up systems. ${ }^{5}{ }^{6} \mathrm{~A}$ recent study across seven European countries found $81 \%$ of men had some unmet supportive care needs and highlighted a lack of support for ongoing symptoms and concerns after treatment. ${ }^{12}$ Prostate Cancer UK has also published the findings from a survey of its members ${ }^{11}$ that shows that over a quarter of men felt that the provision of good-quality information and support was not adequate for their needs, and almost one-third reported follow-up care was not sufficient in terms of addressing the side effects of treatment.

Studies have also shown that healthcare professionals involved in secondary (hospital-based) and primary (general practitioner (GP) clinic-based) care see a greater role for primary care in delivering follow-up care, ${ }^{13}$ and that this would be acceptable to men with prostate cancer. ${ }^{6}$ A recent literature review found no significant differences in patient outcomes in primary versus secondary care follow-up for breast and bowel cancer. ${ }^{14}$ However, no studies so far have assessed primary care-based prostate cancer follow-up.

There is good evidence showing that nurses can be very effective in helping with the management of chronic diseases such as depression ${ }^{15}$ and diabetes, ${ }^{16}$ and also cancer follow-up in primary care. ${ }^{17}$ With respect to prostate cancer, hospital nurse-led follow-up for radiotherapy patients has been shown to be effective and cost-effective when compared with routine medical care, ${ }^{18}$ and a recent American trial indicated that a hospital nurse-led intervention resulted in lasting improvements in the quality of life of men with prostate cancer. ${ }^{19}$

A recent review concluded that promoting selfmanagement by empowering men with prostate cancer to help themselves, is an effective way to address the problems experienced by them. ${ }^{20}$ For example, in the case of men experiencing urinary problems, there is evidence to support the use of pelvic floor muscle exercises and bladder retraining, as well as drug treatments, to improve symptoms. $^{21} \mathrm{~A}$ recent clinical trial from Denmark explored the effects of multidisciplinary (nurse/physical therapist delivered) rehabilitation in patients with prostate cancer who had received radiotherapy and hormone therapy, and found improvements in urinary and hormonal symptoms and physical functioning. ${ }^{22}$ In cases where symptoms cannot be improved, properly identifying incontinence and appropriate use of continence aids may improve quality of life. For some men with sexual dysfunction, drug treatment can help, and recent guidance from the British Society for Sexual Medicine ${ }^{23} 24$ highlights the importance of appropriate prescribing and adequate education to maximise positive outcomes. For men where drug or other therapy does not help the nurse will aim to help them to come to terms with the problem and, for example, help them to discuss the issue with their partner and find other ways of being intimate. Evidence also supports the use of pharmacological treatments for hot flushes experienced by men receiving hormone treatment. ${ }^{25}$

This background evidence has therefore led us to plan a nurse-led intervention delivered in primary care and designed to offer tailored support to men with prostate cancer. Our intervention will be underpinned by the best available evidence regarding the management of physical and psychological problems.

This pilot trial aims to test the acceptability of the intervention, the randomisation procedure and the study measures, and will provide the information required for designing a definitive stage III randomised controlled trial to determine if this model of prostate cancer follow-up care can improve prostate-related quality of life and be cost-effective.

\section{METHODS AND ANALYSIS \\ Design}

This is a two-phase study (see online supplementary appendix 1 for study flow chart). In phase 1 we will conduct a survey of men who are between 9 and 24 months after a prostate cancer diagnosis. Men who participate in the survey, and who are identified by their questionnaire responses as having current problems with urinary, bowel, sexual or hormone-related functioning will be eligible to take part in phase 2 .

Phase 2 is the pilot randomised controlled trial. A trial design has been chosen for this phase because ultimately we wish to determine if a nurse-led intervention delivered in primary care to prostate cancer survivors, in addition to usual care, is effective and cost-effective in improving prostate-related quality of life, compared with usual care alone. Following the Medical Research Council (MRC) guidance $^{26}$ for evaluating complex 
interventions (in this case our nurse-led intervention), a pilot trial/feasibility study is essential for determining whether or not a large trial, which would definitively answer our research question, is feasible and warranted and, if so, to inform the design and sample size for such a trial.

\section{Target group}

Phase 1

For phase 1 we will target men with prostate cancer who are 9-24 months postdiagnosis who have undergone treatment and whose prostate-specific antigen (PSA) values remain stable (surgical patients: $\leq 0.2 \mathrm{ng} / \mathrm{mL}$, radiotherapy patients: $\leq 2 \mathrm{ng} / \mathrm{mL}$ and hormone therapy patients: $\leq 10 \mathrm{ng} / \mathrm{mL}$ ), and men who are on active surveillance.

\section{Phase 2}

For phase 2 men who identify themselves in the phase 1 questionnaire as having problems ('small', 'moderate' or 'big') with urinary, bowel, sexual or hormonal functioning by their responses to the Expanded Prostate cancer Index Composite (EPIC) ${ }^{27}$ will be eligible to participate. Men with a phase 1 Hospital Anxiety and Depression Scale (HADS) ${ }^{28}$ indicating probable depression $(>10)$ are excluded from phase 2 .

\section{Setting}

For phase 1, patients will be recruited through Oxford University Hospitals Trust and Cambridge University Hospitals NHS Foundation Trust. The intervention and follow-up (phase 2) will take place in primary care.

\section{Recruitment of patients}

\section{Phase 1}

Research nurses will identify a random sample of eligible men from hospital databases, stratified by treatment group, and these men will be invited to participate in the study by an invitation letter from the consultant urologist/oncologist in the participating centre. The invitation will be accompanied by a study information sheet and a questionnaire with reply paid envelope. The target group will include men who are already discharged to primary care, as well as those still being seen in secondary care. At the end of the questionnaire, these men will be asked if they would be interested to receive further information about a study which will test the usefulness of nurse-led follow-up care provided in primary care, and if so to provide their contact details. Recruitment will take place over a 9-month period.

\section{Phase 2}

Men who express interest in phase 2, and who identify problems with urinary, bowel, sexual or hormonal functioning on their completed phase 1 questionnaire will be sent detailed information about the trial. This will be followed a week later by a phone call from the study coordinator to discuss the pilot trial in more detail and answer any questions. For men who are still interested in taking part, the study coordinator will seek consent for randomisation over the telephone (this call will be recorded, and will follow a structured script). For practical reasons, we plan to seek consent by phone rather than face-to-face as these men will be dispersed around Oxfordshire and Cambridgeshire and will not necessarily be attending a hospital clinic during the recruitment period.

Men with a HADS score indicating probable depression $(>10)$ are not eligible for phase 2. However, if the patient has provided his contact details (in expressing an interest in participating in phase 2), a letter will be sent from the study clinical lead to the patient advising that questionnaire scores indicate the possibility of depression and that the patient may want to consider seeing his GP for further assessment and support.

\section{Randomisation}

Men who consent will be randomised by a study administrator (who has had no contact with patients nor been involved in contacting them), using a computerised random number generator, to receive either the nurse-led intervention in addition to usual care, or usual care alone. Randomisation will be stratified by treatment type and by study recruitment centre.

\section{Intervention}

The intervention is a nurse-delivered psychoeducational intervention, based on a self-management approach of helping men with prostate cancer to help themselves, ${ }^{29}$ and underpinned by Bandura's Social Cognitive Theory. ${ }^{30}$ Self-efficacy theory is an important component of Bandura's theory which suggests a strong interrelation between an individual's behaviour, environment and cognitive factors. Through self-reflection people make sense of their experiences, explore their own $\operatorname{cog}$ nitions and self-beliefs, engage in self-evaluation and alter their thinking and behaviour accordingly. Bandura contends that self-efficacy beliefs-people's judgements of their capabilities to organise and execute courses of action required to attain designated types of outcomesare central to well-being; unless people believe their actions can produce the desired outcomes they have little incentive to act or to persevere in the case of difficulties. This applies to the problems men experience after prostate cancer treatment in the following manner.

The nurse in our study will be providing tailored information, advice and support, to help men with prostate cancer help themselves either to improve symptoms, or cope with symptoms that cannot be improved. The components of the intervention can be divided into four domains: Understanding the context of prostate cancer treatment; Eliciting needs; Self-management and behavioural activation and Cognitive restructuring (table 1).

Study nurses will receive an intensive initial 2 days of training, followed by a further day to assess intervention provision and fidelity of intervention delivery. In our 


\begin{tabular}{|c|c|c|}
\hline Domain & Details of intervention & Timing and delivery \\
\hline $\begin{array}{l}\text { Understanding the context of } \\
\text { prostate cancer treatment }\end{array}$ & $\begin{array}{l}\text { Determining the impact of diagnosis and treatment } \\
\text { for the individual } \\
\text { Exploring unmet needs }\end{array}$ & $\begin{array}{l}\text { At initial assessment through } \\
\text { discussion using phase } 1 \text { questionnaire } \\
\text { to inform discussion }\end{array}$ \\
\hline Eliciting needs & $\begin{array}{l}\text { Exploring existing symptoms (eg, incontinence, } \\
\text { frequency, bowel problems, sexual function and } \\
\text { fatigue) }\end{array}$ & $\begin{array}{l}\text { Ongoing assessment, focusing on the } \\
\text { areas that mostly affect individual's } \\
\text { quality of life }\end{array}$ \\
\hline $\begin{array}{l}\text { Self-management and } \\
\text { behavioural activation }\end{array}$ & $\begin{array}{l}\text { Goal setting } \\
\text { - Behavioural management, pelvic floor exercises } \\
\text { and bladder retraining techniques } \\
\text { - Weight loss and exercise management } \\
\text { - Appropriate further referral }\end{array}$ & $\begin{array}{l}\text { At initial assessment with } \\
\text { reinforcement at follow-up } \\
\text { appointments }\end{array}$ \\
\hline Cognitive restructuring & $\begin{array}{l}\text { Identifying specific situations or thought patterns } \\
\text { that cause distress to the individual (eg, waiting for } \\
\text { prostate-specific antigen results; thoughts of not } \\
\text { being a man) } \\
\text { Offering tailored support for example, information, } \\
\text { motivation techniques and appropriate further } \\
\text { referral }\end{array}$ & At follow-up \\
\hline
\end{tabular}

study, the study nurses will be either practice nurses or primary care research nurses (who may have varied clinical backgrounds). The intervention will be delivered by an initial face-to-face appointment (30-45 min) with men with prostate cancer. Appointments will be conducted in the patient's own GP clinic wherever possible, or alternatively in the nearest available Primary Care Research Network (PCRN) practice. The nurse will be given a copy of the completed phase 1 questionnaire which will allow prior identification of the likely main issues, and will prompt further assessment and discussion at the appointment. If urinary functioning is a problem, the patient will be sent a frequency/volume chart to complete prior to the appointment, and will be asked to bring a urine sample to the appointment. The nurse will follow an intervention manual which has been developed for the study, and draws on the best available evidence for the management of urinary, bowel, sexual and hormonal functioning problems. In some cases referral to the GP, secondary care or other support services will be appropriate. Further nurse contact with the patient will be individualised, according to need. For example, patients offered advice and information on pelvic floor exercises to help manage urinary incontinence will be followed up to check progress with the exercises, and patients prescribed drugs for erectile dysfunction will be followed up to monitor progress and reinforce advice given. We anticipate patients will need, on average, two shorter follow-up appointments. The frequency, setting and content of these contacts will be recorded by the nurse, and communicated to the patient's GP and consultant (if applicable). At 6 months the nurse will conduct a routine telephone follow-up appointment with all patients for monitoring and support.
Although the study will use trained research nurses, it is intended that, with appropriate education and training, the intervention could be delivered routinely in primary care.

Routine PSA monitoring of the prostate cancer will continue to be overseen by the responsible clinician and will not be part of the intervention.

\section{Usual care}

Usual follow-up care is poorly defined and variable across the UK: previous and updated National Institute of Health and Care Excellence (NICE) guidance on the management of men with prostate cancer recommends care moves outside the hospital setting after at least 2 years for men who are stable, but provides little detail with respect to the specifics of follow-up care. ${ }^{21} 31$ Usual care differs from our intervention in the following ways:

- Generally based in secondary care for $3-5$ years following completion of treatment.

- Often medically focused for example, PSA level, tumour status and medical control of side effects.

- Not based on any formal needs assessments, or tailored to meet the specific needs of individuals.

- Usually very little attention paid to psychological issues.

Usual follow-up care received by study participants will be carefully recorded throughout the study period. No type of care or intervention received as part of usual care prohibits inclusion in the trial. The phase 1 questionnaire will collect information on care received to date. Use of a health service diary questionnaire during the intervention and follow-up period will capture usual care during this phase of the study. 


\section{Sample size}

We aim to recruit 300 men to phase 1 of the study, and 80 men drawn from the phase 1 cohort to the phase 2 pilot trial. Both recruiting centres see in excess of 500 new patients per year. Assuming approximately $50 \%$ of men in phase 1 will report an ongoing problem, ${ }^{32}$ and $60 \%$ of these will consent to be randomised into the pilot trial, we hope to be able to recruit approximately 80 men to the pilot trial (40 to each arm). This will allow us to recruit approximately 8-10 men from each treatment group (radical prostatectomy, radiotherapy, hormone therapy and active surveillance) to the pilot trial and will enable us to address the trial objectives.

\section{Study measures}

Phase 1

The questionnaire will collect information on diagnosis, treatment and current follow-up care. Satisfaction with care will also be assessed, using a series of questions developed for this study. The questionnaire will also use validated tools to measure prostate-related quality of life: the EPIC-26 which assesses urinary, bowel, sexual, hormonal function and bother ${ }^{27}$; HADS to assess psychological morbidity ${ }^{28}$; Supportive Care Needs Survey -34-items Short Form to assess unmet need ${ }^{33}$; EuroQoL EQ-5D to assess health-related quality of life ${ }^{34}$ and the Lorig Scale for self-efficacy, ${ }^{35}$ extended to include cancer-specific questions developed by Foster et al, Macmillan Cancer Survivorship Research Group, University of Southampton (C. F. Foster, University of Southampton, personal communication 2013). Standard sociodemographic details will also be collected.

\section{Phase 2}

A follow-up questionnaire administered at 7 months will repeat the measures used in phase 1 . The questionnaire to the intervention group will also seek their views on the intervention itself. During the intervention phase of the study both groups will be asked to complete a health service use questionnaire which will be used to identify patient-specific resource use data and any out-of-pocket costs directly attributable to the patient.

\section{Interviews}

The experiences and views of the research nurses delivering the intervention $(n=5)$, and a sample of GPs $(n=5)$ on the intervention and participation in the study will be sought, as well as a subsample of men in the intervention group $(n=20)$. These structured interviews will be conducted by the study coordinator by telephone. Interviews will be recorded and transcribed.

\section{Data management procedures}

Completed questionnaires and patient identifiable data will be stored separately in locked cabinets, linked by unique ID. Transmission of data to the study nurses in primary care will use secure, encrypted NHS.net email, secure fax protocol or registered post. To minimise transmission of patient data, nurses will use an anonymised identifier including initials and study ID for all study-related paperwork. A bespoke study database will be used to store and manage information related to the study, with access restricted to key study personnel.

Clear formatting, white space and consistent check box style coded answer format have been incorporated into the questionnaire design to maximise quantity and quality of returns, as well as ease of data entry. Quality of data entry will be further enhanced by the use of dropdown lookup lists and field validation rules. All data will be double entered.

At the end of the study, all study data will be stored securely for a period of at least 2 years after publication of results, at which point all person-identifiable information will be separated out and destroyed securely. The anonymised data will be retained for a minimum of 10 years total.

\section{Phase 1 questionnaire analysis}

A descriptive analysis of phase 1 data will be undertaken looking at differences in prostate-related quality of life, unmet needs, psychological morbidity and self-efficacy between treatment groups and age groups. Estimates of prevalence of urinary, sexual, bowel and hormonerelated problems in prostate cancer survivors will be obtained and presented with their associated 95\% CIs (based on normal approximations or exact methods if estimates are close to 0 or 1 ).

\section{Phase 2 trial analysis}

For preliminary analysis of effectiveness we will estimate the difference in quality of life change between the groups (point estimates and 95\% CIs), and will use analysis of covariance to adjust for baseline levels of the outcome measure and other baseline characteristics such as time since diagnosis. Analysis will be conducted on an intention-to-treat basis. The incremental costs of the nurse-led intervention compared with usual care will be estimated. Patient diaries and questionnaires will be used to identify patient-specific resource use data and any out-of-pocket costs directly attributable to the patient. The primary outcome measure for the costeffectiveness analysis will be the EuroQol health-related quality of life (EQ-5D-5L) questionnaire. The timing of responses to and recall of events for the patient diaries and EQ-5D will be explored. A thematic analysis of interview data will be undertaken. ${ }^{36}$

\section{Service user involvement}

The study has been discussed in detail with the Thames Valley Consumer Research Partnership. Two user representatives from the National Cancer Research Institute (NCRI) Consumer Liaison Group have subsequently joined the study management group. They have been consulted regarding the content of the intervention package, the final set of outcome measures to be used for the study, the patient materials and the recruitment 
process, and will contribute to all stages of the study, including input into the final study report, dissemination of the study findings and development of a future trial proposal.

\section{Potential problems and contingency plans}

Slower than anticipated recruitment: we have been conservative in our estimates of the number of eligible men and are confident that we can reach our accrual target. However, we will monitor recruitment rates on a monthly basis and adopt alternative strategies if necessary.

\section{ETHICS AND DISSEMINATION}

We do not envisage any major ethical issues with this study, and full ethics and governance permission has been granted. Any protocol modifications will be submitted for ethics approval. The study uses standard assessments and interventions that have already been tested. Any drug prescribing will be undertaken by the patient's GP who will have access to their complete medical history. As the clinical and cost-effectiveness of the intervention is unknown, we believe randomising participants to the intervention or usual care is warranted. If the intervention appears to be successful following the pilot we will write to men in the usual care arm suggesting that if they have any ongoing prostate-related quality of life issues that they may wish to contact their GP practice or hospital team to discuss. We do not have sufficient resources to offer men in the control group the intervention at the end of the study. For men in the intervention group we will provide a summary of the intervention to the GP and consultant and identify any ongoing areas of need which will require further input. If the research nurse(s) uncover issues they do not feel equipped to deal with, support will be provided by PR (study co-investigator) assisted by other clinicians involved with the study, and nurses will be trained to alert the patient's GP if there are any concerns. More complex situations, for example withholding consent to divulge, will be discussed with nurses through clinical supervision.

All data will be held securely in accordance with Data Protection Regulations. Patient confidentiality will be maintained at all times. Findings will be widely disseminated in peer-reviewed journals, at conferences, through user networks, and to policy makers and relevant clinical groups. Participants in the trial phase will be offered the option to receive a summary of the results once the study is complete.

\section{DISCUSSION}

This protocol was developed to address a recognised need to improve the quality of care men receive in the period following diagnosis and initial treatment of prostate cancer. Prostate cancer is now the most common cancer in UK men. Incidence and survival rates are rising, resulting in a large and growing number of men living with prostate cancer. It is imperative we maximise the quality of care provided to these men.

While it is known that men with prostate cancer often experience physical, psychological and social problems, we do not have good data on how common these problems are in UK men who are beyond initial treatment, and what their unmet needs are at this time. Phase 1 of this study will begin to address this gap in knowledge.

Phase 2 will offer and pilot test a nurse-led intervention based in primary care. Our intervention will identify and address needs on an individual basis, and will promote self-management. This fits well with the approach being recommended by the UK National Cancer Survivorship Initiative whereby following initial treatment patients are placed into three categories (low, moderate and high) according to their risk of the cancer recurring and the risk of any other complications. Most patients will be in the low-risk category and placed in a supported self-management programme in the community, with access to secondary care if required. In one of the study areas practice nurses have been trained to deliver the intervention and the study will provide important information on the possibility of incorporating this type of approach to cancer follow-up within routine primary care.

Success criteria for the pilot trial are:

- Participation rate of $\geq 50 \%$ for phase 1 questionnaire.

- $>30 \%$ of questionnaire respondents who are eligible for the pilot trial willing to be randomised.

- Acceptable response to follow-up questionnaires $(\geq 50 \%)$.

- Acceptability of intervention to men and health professionals demonstrated (questionnaire and interview data).

- Potential usefulness of intervention to men demonstrated (quantitative and qualitative data).

If these criteria are not met we will still gain useful information on changes needed to the design of the study, or may learn that a full-scale trial is not warranted.

Strengths of the protocol are that it addresses a recognised gap in the care of men with prostate cancer, and that it promotes a model of care consistent with that being recommended by the UK's National Cancer Survivorship Initiative. ${ }^{37}$ Importantly, the study also incorporates a health economics strand. The research team is multidisciplinary and the project is multicentre.

Potential challenges lie in identifying eligible men, achieving good recruitment rates, retaining study nurses over the intervention period, and taking any other relevant health initiatives for men with prostate cancer which may occur over the study period into account.

Author affiliations

${ }^{1}$ Department of Clinical Health Care, Oxford Brookes University, Oxford, UK ${ }^{2}$ Department of Primary Health Care, University of Oxford, Oxford, UK

${ }^{3}$ Nuffield Department of Surgical Sciences, Oxford University Hospitals Trust, Oxford, UK

${ }^{4}$ Department of Surgical Oncology, Cambridge University Hospitals Trust, Cambridge, UK 
${ }^{5}$ Department of Public Health and Primary Care, University of Cambridge, Cambridge, UK

${ }^{6}$ Division of Health and Social Care, University of Surrey, Guildford, UK ${ }^{7}$ Health Economics Research Unit, University of Oxford, Oxford, UK ${ }^{8}$ Centre for Population Health Sciences, University of Edinburgh, Edinburgh, UK ${ }^{9}$ North Wales Centre for Primary Care Research, Bangor University, Wrexham, UK

${ }^{10}$ Service User Representatives, UK

Contributors EW, PR, DW, RN, CC and CW conceived of the study. EW and $\mathrm{PR}$ developed the initial protocol. All authors contributed to refinement of this protocol. EW is the grant holder. DN, CK, FH, PS and SR advised on recruitment from secondary care sites. SF, EW, PR and EF led the development of the nurse intervention and associated training and materials. CW, RN, DW, CC and FMW advised on delivering the intervention in a primary care setting. RP provided guidance on sample size and will lead statistical analyses for the study. JW advised on data requirement for health economics, and undertook these analyses. HB and MM provided user input to the design, study information and questionnaires. EF oversaw the day-to-day implementation of the study according to the protocol. All authors approved the final manuscript.

Funding This study is funded by a grant from Prostate Cancer UK (grant number PG10-09-SV)

Competing interests None.

Ethics approval Oxford Research Ethics committee A (12/SC/0500).

Provenance and peer review Not commissioned; peer reviewed for ethical and funding approval prior to submission.

Open Access This is an Open Access article distributed in accordance with the Creative Commons Attribution Non Commercial (CC BY-NC 4.0) license, which permits others to distribute, remix, adapt, build upon this work noncommercially, and license their derivative works on different terms, provided the original work is properly cited and the use is non-commercial. See: http:// creativecommons.org/licenses/by-nc/4.0/

\section{REFERENCES}

1. Cancer Research UK Statistical Information Team. Prostate Cancer Key Facts. [Web page] Cancer Research UK; 2013 [updated January 2013; cited December 2013]. http://www.cancerresearchuk. org/cancer-info/cancerstats/keyfacts/prostate-cancer/

2. Maddams J, Utley M, Møller H. Projections of cancer prevalence in the United Kingdom, 2010-2040. Br J Cancer 2012;107:1195-202.

3. Boberg EW, Gustafson DH, Hawkins RP, et al. Assessing the unmet information, support and care delivery needs of men with prostate cancer. Patient Educ Couns 2003;49:233-42.

4. Lintz K, Moynihan C, Steginga S, et al. Prostate cancer patients' support and psychological care needs: survey from a non-surgical oncology clinic. Psychooncology 2003;12:769-83.

5. O'Brien R, Rose P, Campbell C, et al. "I wish l'd told them": a qualitative study examining the unmet psychosexual needs of prostate cancer patients during follow-up after treatment. Patient Educ Couns 2011;84:200-7.

6. O'Brien R, Rose PW, Campbell C, et al. Experiences of follow-up after treatment in patients with prostate cancer: a qualitative study. BJU Int 2010;106:998-1003.

7. Ream E, Quennell A, Fincham L, et al. Supportive care needs of men living with prostate cancer in England: a survey. Br J Cancer 2008;98:1903-9.

8. Sanda MG, Dunn RL, Michalski J, et al. Quality of life and satisfaction with outcome among prostate-cancer survivors. N Engl J Med 2008;358:1250-61.

9. Smith DP, King MT, Egger S, et al. Quality of life three years after diagnosis of localised prostate cancer: population based cohort study. BMJ 2009;339:b4817

10. Taylor KL, Luta G, Miller AB, et al. Long-term disease-specific functioning among prostate cancer survivors and noncancer controls in the prostate, lung, colorectal, and ovarian cancer screening trial. $J$ Clin Oncol 2012;30:2768-75.

11. Prostate Cancer UK. Men's views on quality care in prostate cancer -what does quality care mean for men with prostate cancer? National Survey Report, 2012.

12. Cockle-Hearne J, Charnay-Sonnek F, Denis L, et al. The impact of supportive nursing care on the needs of men with prostate cancer: a study across seven European countries. Br J Cancer 2013;109:2121-30.

13. Watson EK, O'Brien R, Campbell C, et al. Views of health professionals on the role of primary care in the follow-up of men with prostate cancer. Fam Pract 2011;28:647-54

14. Lewis RA, Neal RD, Williams $\mathrm{NH}$, et al. Follow-up of cancer in primary care versus secondary care: systematic review. $\mathrm{Br} J$ Gen Pract 2009;59:e234-47.

15. Gilbody S. Review: disease management programmes improve detection and care of people with depression. Evid Based Ment Health 2004;7:80.

16. Renders C, Valk G, Griffin S, et al. Interventions to improve the management of diabetes mellitus in primary care, outpatient and community settings. Cochrane Database Syst Rev 2000;4:CD001481.

17. Lewis R, Neal RD, Williams NH, et al. Nurse-led vs. conventional physician-led follow-up for patients with cancer: systematic review. J Adv Nurs 2009;65:706-23.

18. Faithfull S, Corner J, Meyer L, et al. Evaluation of nurse-led follow up for patients undergoing pelvic radiotherapy. Br J Cancer 2001;85:1853-64.

19. Giesler RB, Given B, Given CW, et al. Improving the quality of life of patients with prostate carcinoma. Cancer 2005;104:752-62.

20. Cockle-Hearne J, Faithfull S. Self-management for men surviving prostate cancer: a review of behavioural and psychosocial interventions to understand what strategies can work, for whom and in what circumstances. Psychooncology 2010;19:909-22.

21. National Institute for Health and Clinical Excellence. Prostate cancer: diagnosis and treatment (CG175) [guideline] January 2014. http:// guidance.nice.org.uk/CG175/NICEGuidance/pdf/English Last updated 6 February 2014, (accessed 28 Feb 2014).

22. Dieperink K, Johansen C, Hansen S, et al. The effects of multidisciplinary rehabilitation: $\mathrm{RePCa}-\mathrm{a}$ randomised study among primary prostate cancer patients. Br J Cancer 2013;109:3005-13.

23. Hackett G, Kell P, Ralph D, et al. British Society for Sexual Medicine guidelines on the management of erectile dysfunction. J Sex Med 2008;5:1841-65.

24. British Society for Sexual Medicine. Guidelines on the Management of Erectile Dysfunction. [guideline] Fisherwick, Staffordshire, BBSSM, 2013. http://www.bssm.org.uk/downloads/BSSM ED Management_Guidelines_2013.pdf (accessed Feb 2014).

25. Loprinzi C, Dueck A, Khoyratty B, et al. A phase III randomized, double-blind, placebo-controlled trial of gabapentin in the management of hot flashes in men. Ann Oncol 2009;20:542-9.

26. Craig $\mathrm{P}$, Dieppe $\mathrm{P}$, Macinture $\mathrm{S}$, et al:; for the Medical Research Council. Developing and evaluating complex interventions: new guidance. London: Medical Research Council, 2008.

27. Szymanski KM, Wei JT, Dunn RL, et al. Development and validation of an abbreviated version of the expanded prostate cancer index composite instrument for measuring health-related quality of life among prostate cancer survivors. Urology 2010;76:1245-50.

28. Zigmond AS, Snaith RP. The hospital anxiety and depression scale. Acta Psychiatr Scand 1983;67:361-70.

29. de Silva D. Helping people help themselves. The Health Foundation, 2011. http://www.health.org.uk/publications/evidence-helping-peoplehelp-themselves/ (accessed Feb 2014).

30. Bandura A. Self-efficacy: toward a unifying theory of behavioral change. Psychol Rev 1977;84:191.

31. National Institute for Health and Clinical Excellence. Prostate cancer diagnosis and treatment (Guidance 58) [guideline, superseded]. NICE, 2008. http://www.nice.org.uk/cg58 (accessed Nov 2013), file no longer available.

32. Wei JT, Dunn RL, Sandler HM, et al. Comprehensive comparison of health-related quality of life after contemporary therapies for localized prostate cancer. J Clin Oncol 2002;20:557-66.

33. Boyes A, Girgis A, Lecathelinais C. Brief assessment of adult cance patients' perceived needs: development and validation of the 34-item Supportive Care Needs Survey (SCNS-SF34). J Eval Clin Pract 2009;15:602-6.

34. EuroQol Group. EuroQol-a new facility for the measurement of health-related quality of life. Health Policy (Amsterdam, Netherlands) 1990;16:199.

35. Lorig KR. Effect of a self-management program on patients with chronic disease. Eff Clin Pract 2001;4:256-62.

36. Braun V, Clarke V. Using thematic analysis in psychology. Qual Res Psychol 2006;3:77-101.

37. Department of Health, Macmillan Cancer Support \& NHS Improvement. Living with \& beyond cancer: taking action to improve outcomes. [Report]. Department of Health, 2013. http://www.ncsi org.uk/wp-content/uploads/Living-with-and-beyond-2013.pdf (accessed Feb 2014) 